\title{
Augmenting Adaptive Approach to Control of Flexible Systems
}

\author{
Anthony J. Calise, ${ }^{*}$ Bong-Jun Yang, ${ }^{\dagger}$ and James I. Craig ${ }^{\ddagger}$ \\ Georgia Institute of Technology, Atlanta, Georgia 30332
}

\begin{abstract}
This paper describes an approach for augmenting a linear controller design with a neural-network-based adaptive element. The basic approach involves formulating an architecture for which the associated error equations have a form suitable for applying existing results for adaptive output feedback control of nonlinear systems. The approach is applicable to non-affine, nonlinear systems with both parametric uncertainties and unmodelled dynamics. The effect of actuator limits are treated using control hedging. The approach is particularly well suited for control of flexible systems subject to limits in control authority. Its effectiveness is tested on a laboratory experiment consisting of a three-disk torsional pendulum system, including control voltage saturation and stiction.
\end{abstract}

\section{Introduction}

$\mathbf{T}$ HIS paper describes an approach for augmenting a linear controller design with a neural-network (NN)-based adaptive element. Previous adaptive output feedback control approaches have been applied within a control architecture that uses an inverting type of controller for the nonadaptive portion of the control system. ${ }^{1,2}$ Considering that the vast majority of controllers are locally linear controllers, it would be highly desirable to retrofit such systems with an adaptive element, rather than to replace them with an inverting controller. In particular, within the aircraft and automobile industries there is a legacy of experience with existing control system architectures, and these industries would much prefer to augment their controllers with an adaptive process, rather than replace them with a totally new architecture. This is particularly the case in applications calling for control of flexible systems.

Several attempts to develop a method for adding an adaptive element to an existing controller architecture have recently appeared in the literature. ${ }^{3-9}$ The methods ${ }^{3-6}$ are restricted to state feedback and impose restrictive conditions with respect to properties of the regulated variable and the manner in which the uncertainty affects the plant. For example, they might require that the regulated output has full relative degree (meaning that the number of times the regulated variable must be differentiated before the control appears equals the number of state variables needed to describe the plant dynamics) or that the plant uncertainty is matched (meaning that the uncertainty enters the plant dynamics in the same manner as the control). Because the methods ${ }^{3-7}$ are based on matching the state response of an idealized model with that of the true plant, they cannot be applied to a system of higher order than the model used in the design process. As a consequence, they are not robust to the unmodeled dynamics. The methods in Refs. 8 and 9 use an adaptive technique called input error method ${ }^{10}$ for reconfigurable flight control. It requires, however, that the open-loop system is stable. State feedback is very restrictive, and flexible systems provide a good example in which a state feedback approach is not useful.

The controller architecture proposed in this paper relies on recent developments in the area of nonlinear adaptive output feedback control. ${ }^{1,2}$ It can be applied to a linear controller architecture without

Received 14 May 2003; revision received 18 October 2003; accepted for publication 20 October 2003. Copyright (C) 2003 by the authors. Published by the American Institute of Aeronautics and Astronautics, Inc., with permission. Copies of this paper may be made for personal or internal use, on condition that the copier pay the $\$ 10.00$ per-copy fee to the Copyright Clearance Center, Inc., 222 Rosewood Drive, Danvers, MA 01923; include the code 0731-5090/04 \$10.00 in correspondence with the CCC.

*Professor, School of Aerospace Engineering; anthony.calise@ae.gatech. edu. Fellow AIAA.

Graduate Research Assistant, School of Aerospace Engineering; bongjun_yang@ae.gatech.edu. Student Member AIAA.

†Professor, School of Aerospace Engineering; james.craig@ae.gatech. edu. Senior Member AIAA. any of the restrictions just mentioned. The main restrictions are that the system to be controlled is minimum phase and that the relative degree of the regulated output variable is known at least over the band of frequencies that the plant is regulated. A recent extension to nonminimum phase systems is described in Refs. 11 and 12. Knowledge of relative degree is a fundamental requirement for any robust control system design. In a linear setting, this assumption amounts to saying that the roll off at the crossover frequency is known. If applied absolutely, then it means that the high frequency roll off is known.

A key feature of the design approach developed here is that the order of the plant dynamics need not be known. The unmodeled effects can consist of internal plant dynamics together with the dynamics of external disturbance processes acting on the plant. Within this context we present a new approach to adaptive cancellation for disturbances generated by an exogenous system. We do not require that sinusoidal disturbances are of known frequencies, which has been a typical assumption in output regulation ${ }^{13}$ and disturbance accommodating control. ${ }^{14}$ The approach can also be applied to control of distributed parameter systems, provided that their dynamics can be approximated to sufficient accuracy by a finite dimensional model. In this setting, the order of the model need not be known. It is assumed that a known lower-order approximate model, having the same relative degree as that of the accurate model, is employed in the design of the linear controller. ${ }^{15}$

A second important aspect in controller design is the effect of nonlinear actuator characteristics, including saturation. To protect the adaptive process, we employ a method of control hedging $(\mathrm{CH})$. This method is analogous to pseudocontrol hedging, ${ }^{16,17}$ previously developed for the case of state feedback, and in the setting of augmenting an inverting controller design. We derive the form needed to apply this method when augmenting a linear controller and provide a stability analysis.

The paper is organized as follows: First we describe the control system architecture and develop the error equation needed to apply an existing approach to adaptive output feedback augmentation. Next, a summary of the approach that can be used for the adaptive portion of the design is presented. The next section presents results related to observability of the system subject to the external disturbance, which is required for adaptive disturbance cancellation. This is followed by a description of how the method of $\mathrm{CH}$ is incorporated into the overall architecture. The last section describes the controller design and experimental results that have been obtained for a three disk torsional laboratory experiment. A proof of the main theorem concerning adaptation with $\mathrm{CH}$ is contained in the Appendix. For simplicity we have limited the presentation to single-input/singleoutput systems. Extensions to multiple-input/multiple-output systems can be found in Refs. 18 and 19.

\section{Control System Architecture}

Figure 1 depicts the conceptual layout for augmenting a linear controller. The nominal feedback control system consists of the true 


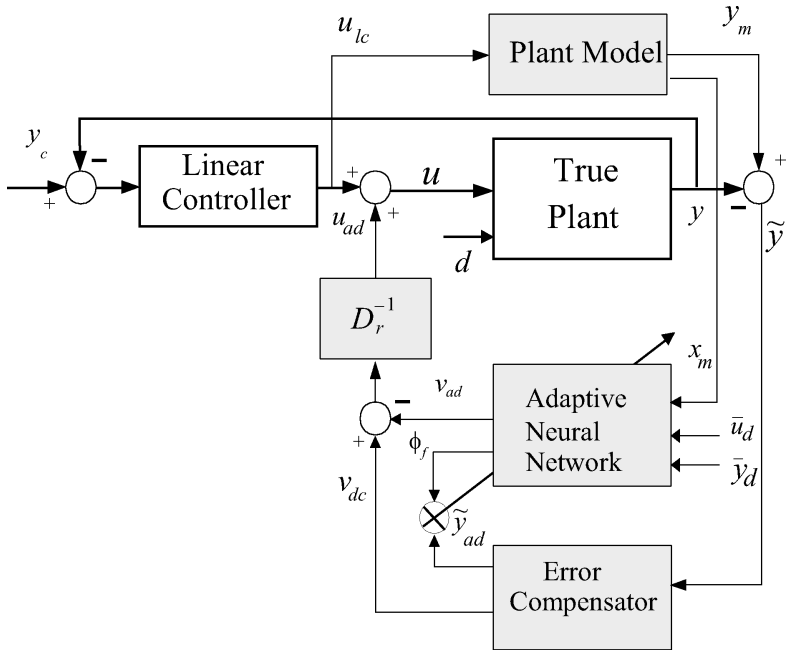

Fig. 1 Adaptive controller architecture.

plant under regulation of a linear controller. It is assumed that the linear controller is designed based on a linear plant model, so that $y_{m}(t)$ tracks $y_{c}(t)$. The linear controller produces the output $u_{\mathrm{lc}}(t)$ that is normally used to regulate the plant. The shaded portion of the diagram highlights the elements to be added to the nominal system. The adaptive process augments the linear controller by adding a signal $u_{\text {ad }}(t)$ to the linear controller output to form the new plant input $u(t)$. The adaptive controller shown is essentially the method of adaptive control design described in Ref. 1. It could just as well be replaced by the method of adaptive control design described in Ref. 2 or any other method of adaptive control design that requires only the output variables to be available for feedback. It is assumed that the plant model is the model used to design the existing controller and that the relative degree of the plant model matches that of the true plant over the bandwidth of interest. Normally, this model is not used to actually control the plant. Here it is used to generate the error signal, $\tilde{y}=y_{m}-y$, which is needed by the adaptive controller. What is important is the form of the equations that describe the dynamics of this error signal $\tilde{y}$ and that it has the correct form for which the theory of adaptive control design is applicable, which will be shown in next section.

\section{Output Tracking Error Equation}

Let the true plant dynamics over the bandwidth of interest be accurately described by

$$
\dot{\boldsymbol{x}}_{p}=\boldsymbol{f}_{p}\left(\boldsymbol{x}_{p}, u, \boldsymbol{d}\right), \quad y=h_{p}\left(\boldsymbol{x}_{p}, \boldsymbol{d}\right)
$$

where $\boldsymbol{x}_{p} \in R^{n_{p}}$ is the state of the system, $u(t) \in R$ is the control variable, $y(t) \in R$ is the regulated output, and $\boldsymbol{d}(t) \in R^{n_{d}}$ is the disturbance. There might be additional outputs that are not regulated but that are available for feedback. These can be included with a slight modification of the overall design approach, and so for simplicity in presentation they are not explicitly treated in the development. We regard the functions $\boldsymbol{f}_{p}$ and $h_{p}$ as uncertain but sufficiently smooth. That is, all needed derivatives exist and are continuous, and if there are unmodelled dynamics then $n_{p}$ is unknown as well.

Assumption 1: The system (1) has known relative degree equal to $r$.

Assumption 2: The system (1) is globally exponentially minimum phase.

Remark 1: In a completely linear setting, it can be shown that the closed-loop eigenvalues of the plant model when regulated by the linear controller, together with zeros for the system in Eq. (1), constitute the eigenvalues of the zero dynamics for the closed-loop system depicted in Fig. 1.

The bounded disturbance vector $\boldsymbol{d}(t)$ evolves according to its own dynamics defined by

$$
\dot{x}_{d}=f_{d}\left(x_{d}\right), \quad \boldsymbol{d}=\boldsymbol{h}_{d}\left(\boldsymbol{x}_{d}\right)
$$

where $\boldsymbol{x}_{d}(t) \in R^{n_{d}}$. The functions $\boldsymbol{f}_{d}$ and $\boldsymbol{h}_{d}$ are sufficiently smooth, but also uncertain, because even though disturbances have finite bandwidth they are usually unknown functions of time. The augmented system consisting of the plant and disturbance dynamics can now be expressed as

$$
\begin{gathered}
\dot{\boldsymbol{x}}=\left[\begin{array}{c}
\dot{\boldsymbol{x}}_{p} \\
\dot{\boldsymbol{x}}_{d}
\end{array}\right]=\left[\begin{array}{c}
\boldsymbol{f}_{p}\left(\boldsymbol{x}_{p}, u, \boldsymbol{h}_{d}\left(\boldsymbol{x}_{\boldsymbol{d}}\right)\right) \\
\boldsymbol{f}_{d}\left(\boldsymbol{x}_{d}\right)
\end{array}\right]=\boldsymbol{f}(\boldsymbol{x}, u) \\
y=h_{p}\left(\boldsymbol{x}_{p}, \boldsymbol{h}_{d}\left(\boldsymbol{x}_{d}\right)\right)=h(\boldsymbol{x})
\end{gathered}
$$

Assumption 1 implies

$$
\begin{gathered}
y^{(i)}=h_{i}(\boldsymbol{x}), \quad 0 \leq i \leq r-1 \\
y^{(r)}=h_{r}(\boldsymbol{x}, u)
\end{gathered}
$$

that is,

$$
\frac{\partial h_{i}}{\partial u}=0, \quad \forall i \quad 0 \leq i \leq r-1 \quad \text { and } \quad \frac{\partial h_{r}}{\partial u} \neq 0
$$

Assumption 3: The system (3) is locally observable in $R^{n_{p}+n_{d}}$. Let the plant model be described by

$$
\begin{gathered}
\dot{\boldsymbol{x}}_{m}=\boldsymbol{A}_{m} \boldsymbol{x}_{m}+\boldsymbol{B}_{m} u_{\mathrm{lc}}, \quad y_{m}=\boldsymbol{C}_{m} \boldsymbol{x}_{m} \\
y_{m}^{(r)} \triangleq \hat{h}_{r}\left(\boldsymbol{x}_{m}, u_{\mathrm{lc}}\right)=\boldsymbol{C}_{r} \boldsymbol{x}_{m}+D_{r} u_{\mathrm{lc}}
\end{gathered}
$$

where $\boldsymbol{x}_{m} \in R^{m}\left(m \leq n_{p}\right)$ and

$$
\boldsymbol{C}_{r}=\boldsymbol{C}_{m} \boldsymbol{A}_{m}^{r}, \quad D_{r}=\boldsymbol{C}_{m} \boldsymbol{A}_{m}^{r-1} \boldsymbol{B}_{m}
$$

The linear control signal $u_{\mathrm{lc}}$ is the output of the following compensator:

$$
\dot{\boldsymbol{x}}_{c}=\boldsymbol{A}_{c} \boldsymbol{x}_{c}+\boldsymbol{B}_{c}\left(y_{c}-y\right), \quad u_{\mathrm{lc}}=\boldsymbol{C}_{c} \boldsymbol{x}_{c}+D_{c}\left(y_{c}-y\right)
$$

where $\boldsymbol{x}_{c} \in R^{n_{c}}$. The plant model in Eq. (5) regulated by the linear controller in Eq. (7), with the replacement of $y$ by $y_{m}$, results in the following nominal closed-loop system:

$$
\dot{\overline{\boldsymbol{x}}}_{\mathrm{nom}}=\boldsymbol{F} \overline{\boldsymbol{x}}_{\mathrm{nom}}+\boldsymbol{G}_{c} y_{c}(t), \quad y_{\mathrm{nom}}=\boldsymbol{H} \overline{\boldsymbol{x}}_{\mathrm{nom}}
$$

where $\boldsymbol{x}_{\mathrm{nom}} \in R^{m+n_{c}}$ and

$$
\begin{gathered}
\boldsymbol{F}=\left[\begin{array}{cc}
\boldsymbol{A}_{m}-\boldsymbol{B}_{m} D_{c} \boldsymbol{C}_{m} & \boldsymbol{B}_{m} \boldsymbol{C}_{c} \\
-\boldsymbol{B}_{c} \boldsymbol{C}_{m} & \boldsymbol{A}_{c}
\end{array}\right] \\
\boldsymbol{G}_{c}=\left[\begin{array}{c}
\boldsymbol{B}_{m} D_{c} \\
\boldsymbol{B}_{c}
\end{array}\right], \quad \boldsymbol{H}^{T}=\left[\begin{array}{c}
\boldsymbol{C}_{m}^{T} \\
0
\end{array}\right]
\end{gathered}
$$

where $y_{c}(t)$ is a bounded reference command. It is reasonable to assume that the controller in Eq. (7) is designed so that performance specifications are satisfied by the nominal system in Eq. (8). Consequently, $\boldsymbol{F}$ in Eq. (9) is Hurwitz.

Using Eqs. (4-6), the following error dynamics can be derived:

$$
\begin{aligned}
\tilde{y}^{(r)} & =-D_{r}\left(u-u_{\mathrm{lc}}\right)-\Delta\left(\boldsymbol{x}, \boldsymbol{x}_{m}, u\right) \\
& =-D_{r} u_{\mathrm{ad}}-\Delta\left(\boldsymbol{x}, \boldsymbol{x}_{m}, u\right)
\end{aligned}
$$

where $\tilde{y}=y_{m}-y$ and

$$
\Delta\left(\boldsymbol{x}, \boldsymbol{x}_{m}, u\right)=h_{r}(\boldsymbol{x}, u)-\boldsymbol{C}_{r} \boldsymbol{x}_{m}-D_{r} u
$$

It can be seen from Eq. (10) that the goal of $u_{\text {ad }}$ is to stabilize the error dynamics and cancel $\Delta\left(\boldsymbol{x}, \boldsymbol{x}_{m}, u\right)$. The adaptive term in Eq. (10) is expressed as

$$
u_{\mathrm{ad}}=D_{r}^{-1}\left(v_{\mathrm{dc}}-v_{\mathrm{ad}}\right)
$$

where $v_{\mathrm{dc}}$ is the output of a linear controller, with $\tilde{y}$ as its input, that is designed to stabilize the error dynamics in Eq. (10) when 
$\Delta\left(\boldsymbol{x}, \boldsymbol{x}_{m}, u\right)=0$ and $v_{\text {ad }}$ is the output of a NN, whose weights are adapted in a way to guarantee a bounded error response. Substituting Eq. (12) into Eq. (10) results in the final form of the error equation:

$$
\tilde{y}^{(r)}=-v_{\mathrm{dc}}+v_{\mathrm{ad}}-\Delta
$$

From Eqs. (10) and (11), it follows that $\Delta$ depends on $v_{\text {ad }}$ through $u$, whereas $v_{\text {ad }}$ is designed to cancel $\Delta$.

Assumption 4: There exists a fixed point to the equation $v_{\mathrm{ad}}=$ $\Delta\left(\boldsymbol{x}, \boldsymbol{x}_{m}, v_{\mathrm{ad}}\right)$ for all $\boldsymbol{x}, \boldsymbol{x}_{m}$ in the domain of interest.

According to Brouwer fixed-point theorem, ${ }^{20}$ any continuous function with a bounded domain and range contained in the bounded domain must have at least one fixed point. Existence and uniqueness of a fixed point is guaranteed when the mapping $v_{\mathrm{ad}} \rightarrow \Delta$ is a contraction. It can be shown that the map $v_{\text {ad }} \rightarrow \Delta$ is a contraction if and only if the following two conditions are satisfied ${ }^{1}$ :

$$
\operatorname{sign}\left(D_{r}\right)=\operatorname{sign}\left(\frac{\partial h_{r}}{\partial u}\right), \quad\left|\frac{\partial h_{r}}{\partial u}\right| / 2<\left|D_{r}\right|<\infty
$$

These conditions mean that control reversal is not permitted and there is a lower bound on the estimate of the control effectiveness $D_{r}$ of the plant model. A different viewpoint that uses the mean value theorem to eliminate the fixed-point assumption can be found in Refs. 19 and 21.

\section{Adaptive Output Feedback Augmentation}

The approach in Ref. 1 allows for designing the signals $v_{\mathrm{dc}}$ and $v_{\text {ad }}$ in Eq. (13) using only available measurements. The error compensator has two outputs:

$$
\left[\begin{array}{c}
v_{\mathrm{dc}}(s) \\
\tilde{y}_{\mathrm{ad}}(s)
\end{array}\right]=\frac{1}{D_{\mathrm{dc}}(s)}\left[\begin{array}{c}
N_{\mathrm{dc}}(s) \\
N_{\mathrm{ad}}(s)
\end{array}\right] \tilde{y}(s)
$$

where the first output $v_{\mathrm{dc}}$ is designed to stabilize the error dynamics in Eq. (13) and the second output $\tilde{y}_{\mathrm{ad}}$ is a training signal for a $\mathrm{NN}$, which is a linear combination of the error compensator states and its input, that is, the tracking error $\tilde{y}$. With the error compensator in Eq. (15), the error equation given in Eq. (13) results in the following transfer function from $v_{\text {ad }}-\Delta$ to $\tilde{y}_{\text {ad }}$ :

$$
\tilde{y}_{\mathrm{ad}}(s)=\frac{N_{\mathrm{ad}}(s)}{s^{r} D_{\mathrm{dc}}(s)+N_{\mathrm{dc}}(s)}\left(v_{\mathrm{ad}}-\Delta\right) \equiv G(s)\left(v_{\mathrm{ad}}-\Delta\right)
$$

A linearly parameterized $\mathrm{NN}$ is used to approximate $\Delta$ in Eq. (11). It is a universal approximator if a set of basis functions can be selected over a compact domain of approximation. For example, it has been shown that an arbitrary continuous function can be approximated to any desired accuracy on a compact domain using radial basis functions. ${ }^{22}$ The result has been extended to map the uncertainty of an observable plant, generally a function of states and control, from available input/output history. ${ }^{23,24}$ Given $\epsilon>0, \Delta$ can be approximated by a linearly parameterized NN over a compact domain with bounded weights $\boldsymbol{W}$ and a suitable set of basis functions $\phi(\cdot)$ that provide a universal approximation

$$
\Delta=\boldsymbol{W}^{T} \phi(\boldsymbol{\eta})+\varepsilon(\boldsymbol{\eta}), \quad\|\varepsilon(\boldsymbol{\eta})\|<\epsilon
$$

where $\varepsilon(\boldsymbol{\eta})$ is the $\mathrm{NN}$ reconstruction error and $\boldsymbol{\eta}$ is the network input vector

$$
\begin{gathered}
\boldsymbol{\eta}(t)=\left[\begin{array}{llll}
1 & \boldsymbol{x}_{m}(t)^{T} & \bar{u}_{d}^{T}(t) & \bar{y}_{d}^{T}(t)
\end{array}\right]^{T} \\
\bar{u}_{d}^{T}(t)=\left[\begin{array}{llll}
u(t) & u(t-d) & \cdots & u\left(t-\left(n_{1}-r-1\right) d\right)
\end{array}\right]^{T} \\
\bar{y}_{d}^{T}(t)=\left[\begin{array}{llll}
y(t) & y(t-d) & \cdots & y\left(t-\left(n_{1}-1\right) d\right)
\end{array}\right]^{T}
\end{gathered}
$$

where $n_{1} \geq n$ is the length of a sliding window of measurements, $r$ is the relative degree, and $d>0$ is a positive time delay. The output of the adaptive element in Fig. 1 is designed as

$$
v_{\mathrm{ad}}=\hat{\boldsymbol{W}}^{T} \phi(\boldsymbol{\eta})
$$

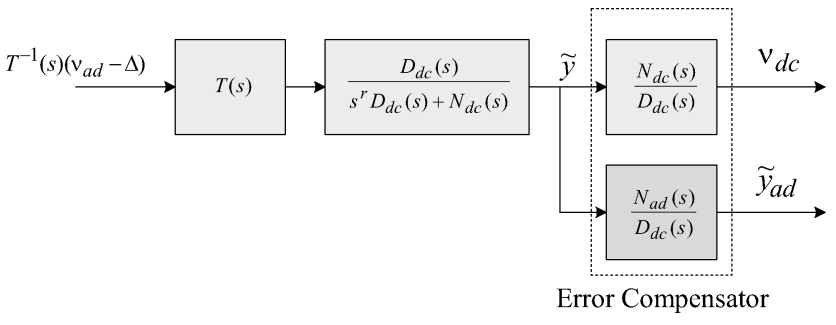

Fig. 2 Block diagram for the error compensator and the SPR filter.

where $\hat{\boldsymbol{W}}$ are estimates of the weights $\boldsymbol{W}$ in Eq. (17) that are adjustable online.

To obtain an adaptation rule dependent only upon available signals, $G(s)$ is required to be strictly positive real (SPR). ${ }^{1} G(s)$ can be made SPR by properly choosing $N_{\text {ad }}(s)$ in case $r=1$. If $r>1$, a stable low-pass filter $T^{-1}(s)$ is introduced so that $G(s) T(s)$ is SPR

$$
\tilde{y}_{\mathrm{ad}}(s)=G(s) T(s)\left[T^{-1}(s)\left(v_{\mathrm{ad}}-\Delta\right)\right]
$$

where the polynomial $T(s)$ is Hurwitz, but can otherwise be freely chosen along with $N_{\text {ad }}(s)$ of the error compensator in Eq. (15).

The filtered NN reconstruction error, $\psi \triangleq T^{-1}(s)\left(v_{\text {ad }}-\Delta\right)$, can be written as follows ${ }^{1}$ :

$$
\psi=\tilde{\boldsymbol{W}}^{T} \boldsymbol{\phi}_{f}+\theta-\varepsilon_{f}
$$

where $\phi_{f}$ and $\varepsilon_{f}$ are the signals $\phi$ and $\varepsilon$, respectively, after being filtered through $T^{-1}(s)$, and $\theta$ is the mismatch term given by

$$
\theta(s)=T^{-1}(s)\left(\tilde{\boldsymbol{W}}^{T} \phi\right)-\tilde{\boldsymbol{W}}^{T} \phi_{f}
$$

that can be bounded as

$$
\|\theta\| \leq \alpha\|\tilde{\boldsymbol{W}}\|_{F}, \quad \alpha>0
$$

where $\tilde{\boldsymbol{W}}=\hat{\boldsymbol{W}}-\boldsymbol{W}$ represents weight deviations from ideal weights $\boldsymbol{W}$. The transfer function from $\psi$ to $\tilde{y}$ is realized as follows (see Fig. 2):

$$
\dot{z}_{e}=\boldsymbol{A}_{e} z_{e}+\boldsymbol{B}_{e} \psi, \quad \tilde{y}=\boldsymbol{C}_{e} z_{e}
$$

The transfer functions from $\tilde{y}$ to $\tilde{y}_{\mathrm{ad}}$ and $v_{\mathrm{dc}}$ are realized as follows:

$$
\begin{gathered}
\dot{\boldsymbol{z}}_{\mathrm{dc}}=\boldsymbol{A}_{\mathrm{dc}} z_{\mathrm{dc}}+\boldsymbol{B}_{\mathrm{dc}} \tilde{y}, \quad \tilde{y}_{\mathrm{ad}}=\boldsymbol{C}_{\mathrm{ad}} z_{\mathrm{dc}}+D_{\mathrm{ad}} \tilde{y} \\
v_{\mathrm{dc}}=\boldsymbol{C}_{\mathrm{dc}} z_{\mathrm{dc}}+D_{\mathrm{dc}} \tilde{y}
\end{gathered}
$$

where $\boldsymbol{A}_{\mathrm{dc}}$ is assumed Hurwitz. Combining Eqs. (24) and (25) leads to the following nonminimal realization for the tracking error dynamics in Fig. 2.

$$
\begin{array}{cc}
\dot{z}=\boldsymbol{A}_{\mathrm{cl}} z+\boldsymbol{B}_{\mathrm{cl}} \psi, & \tilde{y}_{\mathrm{ad}}=\boldsymbol{C}_{\mathrm{cl}} z \\
v_{\mathrm{dc}}=\boldsymbol{C}_{v} z, & \tilde{y}=\boldsymbol{C}_{\tilde{y}} z
\end{array}
$$

where

$$
\begin{gathered}
\boldsymbol{A}_{\mathrm{cl}}=\left[\begin{array}{cc}
\boldsymbol{A}_{e} & 0 \\
\boldsymbol{B}_{\mathrm{dc}} \boldsymbol{C}_{e} & \boldsymbol{A}_{\mathrm{dc}}
\end{array}\right], \quad \boldsymbol{B}_{\mathrm{cl}}=\left[\begin{array}{c}
\boldsymbol{B}_{e} \\
0
\end{array}\right], \quad \boldsymbol{C}_{\mathrm{cl}}=\left[\begin{array}{ll}
D_{\mathrm{ad}} \boldsymbol{C}_{e} & \boldsymbol{C}_{\mathrm{ad}}
\end{array}\right] \\
\boldsymbol{C}_{v}=\left[\begin{array}{ll}
D_{\mathrm{dc}} \boldsymbol{C}_{e} & \boldsymbol{C}_{\mathrm{dc}}
\end{array}\right], \quad \boldsymbol{C}_{\tilde{y}}=\left[\begin{array}{ll}
\boldsymbol{C}_{e} & 0
\end{array}\right]
\end{gathered}
$$

Because the transfer function from $\psi$ to $\tilde{y}_{\text {ad }}$ is SPR, by the MeyerKalman-Yakubovitz lemma, ${ }^{25}$ there exist $Q>0$ and $P>0$ such that

$$
\boldsymbol{A}_{\mathrm{cl}}^{T} P+P \boldsymbol{A}_{\mathrm{cl}}+Q=0, \quad P \boldsymbol{B}_{\mathrm{cl}}=\boldsymbol{C}_{\mathrm{cl}}^{T}
$$

The SPR filter $T^{-1}(s)$ is realized by

$$
\dot{z}_{f}=\boldsymbol{A}_{f} \boldsymbol{z}_{f}+\boldsymbol{B}_{f} \boldsymbol{\phi}, \quad \boldsymbol{\phi}_{f}=\boldsymbol{C}_{f} \boldsymbol{z}_{f}
$$


The SPR filter is designed to be stable, so that for any $Q_{f}>0$ there exist $P_{f}>0$ such that

$$
\boldsymbol{A}_{f}^{T} P_{f}+P_{f} \boldsymbol{A}_{f}+Q_{f}=0
$$

The signals $\phi_{f}$ are used in the following $\mathrm{NN}$ adaptation rule:

$$
\dot{\hat{\boldsymbol{W}}}=-\Gamma_{W}\left(\tilde{y}_{\mathrm{ad}} \phi_{f}+\sigma \hat{\boldsymbol{W}}\right)
$$

where $\Gamma_{W}>0$ is the adaptation gain, defining the learning rate and $\sigma \hat{W}$ is the $\sigma$-modification term. ${ }^{26}$ The procedure for designing the error compensator, the SPR filter $T(s)$, and the stability proof for $\mathrm{NN}$ adaptation law is given in Ref. 1 .

Theorem 1: With assumptions 1-4, the error signals of the system comprised of the dynamics in Eq. (13), together with feedback control law $u=u_{\mathrm{lc}}+D_{r}^{-1}\left(v_{\mathrm{dc}}-v_{\mathrm{ad}}\right)$ and the $\mathrm{NN}$ adaptation rule in Eq. (31), are uniformly ultimately bounded.

Proof: The result follows from the theorems given in Ref. 1 and the fact that Eq. (3) is locally observable.

Theorem 1 guarantees boundedness of the output tracking error $\tilde{y}$ and NN weights $\hat{\boldsymbol{W}}$. Because the linear controller is designed to stabilize the plant model, it immediately follows that if $y_{c}(t)$ and $\tilde{y}(t)$ are bounded, then $e(t)=y_{c}-y$ is bounded. It is also apparent that when $\tilde{y}=0$ we recover the tracking performance associated with the existing controller design, with the plant model substituted for the true plant. In particular, for this idealized setting the disturbances $\boldsymbol{d}(t)$ will be cancelled.

\section{Observability of the Augmented System}

Theorem 2: If the linear system

$$
\dot{x}=A x, \quad y=C x
$$

is observable, where $\boldsymbol{A}=\left.(\partial \boldsymbol{f} / \partial \boldsymbol{x})\right|_{x=x_{0}}$ and $\boldsymbol{C}=\left.(\partial h / \partial \boldsymbol{x})\right|_{x=x_{0}}$, then the nonlinear system in Eq. (3) is locally observable at the point $\boldsymbol{x}_{0}$.

Proof: See Ref. 27, theorem 26.

Define

$$
\begin{aligned}
& \boldsymbol{A}=\left[\begin{array}{cc}
\frac{\partial \boldsymbol{f}_{p}}{\partial \boldsymbol{x}_{p}} & \frac{\partial \boldsymbol{f}_{p}}{\partial \boldsymbol{d}} \frac{\partial \boldsymbol{h}_{d}}{\boldsymbol{x}_{d}} \\
0 & \frac{\partial \boldsymbol{f}_{d}}{\partial \boldsymbol{x}_{d}}
\end{array}\right]_{x=x_{0}}=\left[\begin{array}{cc}
\boldsymbol{A}_{p} & \boldsymbol{B}_{p} \boldsymbol{C}_{d} \\
0 & \boldsymbol{A}_{d}
\end{array}\right]
\end{aligned}
$$

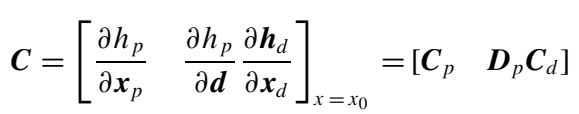

The following theorem establishes a sufficient condition for the local observability of the augmented nonlinear system in Eq. (3).

Theorem 3: Consider the system of matrices defined in Eq. (32) together with the following assumptions: 1) $\left(\boldsymbol{A}_{p}, \boldsymbol{C}_{p}\right)$ observable; 2) $\left(\boldsymbol{A}_{d}, \boldsymbol{C}_{d}\right)$ observable; and 3$)$

$$
\left[\begin{array}{l}
\boldsymbol{B}_{p} \\
\boldsymbol{D}_{p}
\end{array}\right]
$$

has full column rank.

Then the augmented nonlinear system in Eq. (3) is locally observable at $\boldsymbol{x}=\boldsymbol{x}_{0}$ if $\boldsymbol{A}_{p}$ and $\boldsymbol{A}_{d}$ have no common eigenvalues.

Proof: See Appendix.

Condition 2 means that the realization of the disturbance model is locally minimal, and condition 3 means that the realization of the influence that the disturbance has on the plant is locally minimal; therefore, these two conditions are not restrictive. The following corollary shows that, subject to the assumptions in theorem 3, nonlinear systems are almost always observable.

Corollary 1: Under the assumptions stated in theorem 3, the augmented system in Eq. (32) is not observable if and only if $\boldsymbol{A}_{p}$ and $\boldsymbol{A}_{d}$ share a common eigenvalue $\lambda \in C$, and $\overline{\boldsymbol{C}} \boldsymbol{q}_{2}$ lies in the column space of $\left[\begin{array}{lll}\boldsymbol{C}_{p}^{T} & \lambda \boldsymbol{I}_{n_{p}} & -\boldsymbol{A}_{p}^{T}\end{array}\right]^{T}$, where

$$
\overline{\boldsymbol{C}}=\left[\begin{array}{c}
\boldsymbol{D}_{p} \\
-\boldsymbol{B}_{p}
\end{array}\right] \boldsymbol{C}_{d}
$$

and $\boldsymbol{q}_{2}$ is the eigenvector of $\boldsymbol{A}_{d}$ associated with $\lambda$.

Proof: See Appendix.

Theorem 3 taken together with corollary 1 suggests that the adaptive element should be able to cancel the effect of $\boldsymbol{d}(t)$ on $y(t)$, modulo the effect because of to a component of $\boldsymbol{d}(t)$ satisfying a very narrow set of conditions. The generic property of observability further justifies that the observability assumption is not restrictive. ${ }^{27}$

\section{Control Hedging in Adaptive Output Feedback Augmentation}

Pseudocontrol hedging ${ }^{17}$ is introduced to protect an adaptive process, by preventing it from attempting to adapt to selected input nonlinearities (such as those caused by saturation). This is accomplished by modeling and removing the effect of these nonlinearities in the error dynamics. This permits the adaptive process to continue to estimate the modeling error, so that when the process comes out of saturation the correct adaptive control is immediately available. The approach was originally developed for use when augmenting an inverting controller. The implementation of pseudocontrol hedging consists of calculating the difference between a commanded pseudocontrol signal and an estimate of the achievable pseudocontrol. This difference is referred to as the hedge signal because it is subtracted from the dynamics of a command filter. Its implementation in the context of augmenting a linear controller is illustrated in Fig. 3. In this setting the difference is formed at the level of the control signal, rather than the pseudocontrol signal, and so we refer to it as $\mathrm{CH}$, rather than pseudocontrol hedging. The nonlinear input characteristic is defined as

$$
\delta \triangleq g(u), \quad|\delta| \leq \delta_{0}
$$

where $u$ is the commanded control input and $\delta_{0}$ is the control limit. With the input nonlinearity, the dynamics in Eq. (3) are written as

$$
\dot{x}=\boldsymbol{f}(\boldsymbol{x}, g(u)), \quad y=h(\boldsymbol{x}), \quad y^{(r)}=h_{r}(\boldsymbol{x}, g(u))
$$

If $\delta$ is available for feedback, then $\hat{\delta}=\delta$. Otherwise, we assume it is estimated using

$$
\hat{\delta}=\hat{g}(u), \quad|\hat{\delta}| \leq \hat{\delta}_{0}
$$

where $\hat{\delta}_{0}$ is an estimate for $\delta_{0}$. Further we assume that the estimate for the input satisfies a Lipschitz condition. This assumption is required for stability analysis.

Assumption 5: There exists $L>0$ such that $\left\|\hat{g}\left(u_{1}\right)-\hat{g}\left(u_{2}\right)\right\| \leq$ $L\left|u_{1}-u_{2}\right|$ for $\forall u_{1}, u_{2} \in \mathcal{U}$.

Input saturation globally satisfies assumption 5 with $L=1$.

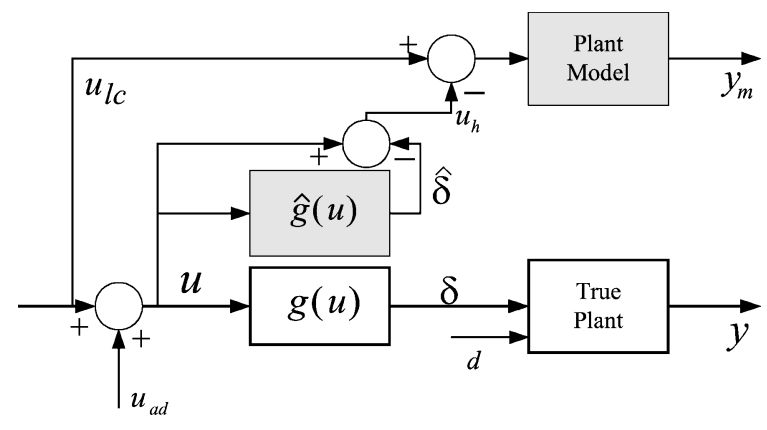

Fig. 3 Implementation of control hedging. 
With $\mathrm{CH}$, the plant model dynamics in Eq. (5) are modified as follows:

$$
\begin{array}{lr}
\dot{\boldsymbol{x}}_{m}=\boldsymbol{A}_{m} \boldsymbol{x}_{m}+\boldsymbol{B}_{m}\left(u_{\mathrm{lc}}-u_{h}\right), & u_{h}=u-\hat{\delta} \\
y_{m}=\boldsymbol{C}_{m} \boldsymbol{x}_{m}, & y_{m}^{(r)}=\boldsymbol{C}_{r} \boldsymbol{x}_{m}+D_{r}\left(u_{\mathrm{lc}}-u_{h}\right)
\end{array}
$$

where

$$
u_{h}=u_{\mathrm{lc}}+D_{r}^{-1}\left(v_{\mathrm{dc}}-v_{\mathrm{ad}}\right)-\hat{g}\left(u_{\mathrm{lc}}+D_{r}^{-1}\left(v_{\mathrm{dc}}-v_{\mathrm{ad}}\right)\right)
$$

The plant model dynamics in Eq. (36) regulated by the linear controller in Eq. (7) can be described by

$$
\dot{\overline{\boldsymbol{x}}}=\boldsymbol{F} \overline{\boldsymbol{x}}+\boldsymbol{G}_{c} y_{c}+\boldsymbol{G}_{c} \tilde{y}-\boldsymbol{G} u_{h}
$$

where $\overline{\boldsymbol{x}}^{T}=\left[\boldsymbol{x}_{m}^{T}, \boldsymbol{x}_{c}^{T}\right]^{T}, \boldsymbol{G}^{T}=\left[\boldsymbol{B}_{m}^{T}, 0^{T}\right]^{T}$. Define the plant model error vector $\boldsymbol{e}_{m} \triangleq \overline{\boldsymbol{x}}_{\text {nom }}-\overline{\boldsymbol{x}}$, then comparing Eq. (38) to Eq. (8) leads to the following plant model error dynamics:

$$
\dot{\boldsymbol{e}}_{m}=\boldsymbol{F} \boldsymbol{e}_{m}-\boldsymbol{G}_{c} \tilde{y}+\boldsymbol{G} u_{h}, \quad \boldsymbol{e}_{m} \in \boldsymbol{\Omega}_{e_{m}} \subseteq R^{m+n_{c}}
$$

Because $\boldsymbol{F}$ is Hurwitz by design, for any $Q_{n}>0$, there exists $P_{n}>0$ such that

$$
\boldsymbol{F}^{T} P_{n}+P_{n} \boldsymbol{F}+Q_{n}=0
$$

With the form of $\hat{h}_{r}$ in Eq. (5) and $\hat{\delta}$ in Eq. (35), $y^{(r)}$ in Eq. (34) can be written as

$$
\begin{aligned}
y^{(r)} & =\hat{h}_{r}\left(\boldsymbol{x}_{m}, \hat{g}(u)\right)+\Delta\left(x, \boldsymbol{x}_{m}, u\right) \\
& =\boldsymbol{C}_{r} \boldsymbol{x}_{m}+D_{r} \hat{\delta}+\Delta\left(\boldsymbol{x}, \boldsymbol{x}_{m}, u\right)
\end{aligned}
$$

where

$$
\Delta\left(\boldsymbol{x}, \boldsymbol{x}_{m}, u\right)=h_{r}(\boldsymbol{x}, g(u))-\boldsymbol{C}_{r} \boldsymbol{x}_{m}-D_{r} \hat{\delta}
$$

Comparing Eqs. (36) and (41) leads to the following output tracking error dynamics:

$$
\tilde{y}^{(r)}=-D_{r} u_{\mathrm{ad}}-\Delta\left(\boldsymbol{x}, \boldsymbol{x}_{m}, u\right)
$$

Using Eq. (12), the output tracking error dynamics in Eq. (10) is finally written as

$$
\tilde{y}^{(r)}=-v_{\mathrm{dc}}+v_{\mathrm{ad}}-\Delta
$$

Note that the error dynamics maintain the same form as in Eq. (13), while the definition of the modeling error $\Delta$ has changed from that in Eq. (11) to that in Eq. (42), which implies that theorem 1 remains valid so long as assumptions 2 and 4 , with $\Delta$ being defined as in Eq. (42), are not violated in the presence of an actuator characteristic.

For stability analysis, we introduce the following definition:

$$
u_{h}^{*}=u_{\mathrm{lc}}+D_{r}^{-1}\left(v_{\mathrm{dc}}-v_{\mathrm{ad}}^{*}\right)-\hat{g}\left(u_{\mathrm{lc}}+D_{r}^{-1}\left(v_{\mathrm{dc}}-v_{\mathrm{ad}}^{*}\right)\right)
$$

which is $u_{h}$ in Eq. (37) when $\mathrm{NN}$ adaptation is exact, that is, $v_{\mathrm{ad}}=v_{\mathrm{ad}}^{*}=\boldsymbol{W} \phi(\boldsymbol{\eta})$. With Eqs. (7) and (25), $u_{h}^{*}$ can be explicitly written as

$$
\begin{aligned}
u_{h}^{*}= & D_{c} y_{c}+J_{1} \overline{\boldsymbol{x}}_{\mathrm{nom}}-J_{1} \boldsymbol{e}_{m}+J_{2} z-D_{r}^{-1} \boldsymbol{W} \boldsymbol{\phi} \\
& -\hat{g}\left(D_{c} y_{c}+J_{1} \overline{\boldsymbol{x}}_{\mathrm{nom}}-J_{1} \boldsymbol{e}_{m}+J_{2} z-D_{r}^{-1} \boldsymbol{W} \boldsymbol{\phi}\right)
\end{aligned}
$$

where $J_{1}=\left[-D_{c} \boldsymbol{C}_{m}, \boldsymbol{C}_{c}\right]$ and $J_{2}=\left[\left(D_{c}+D_{r}^{-1} D_{\mathrm{dc}}\right) \boldsymbol{C}_{e}, D_{r}^{-1} \boldsymbol{C}_{\mathrm{dc}}\right]$. Note that $u_{h}^{*}$ depends on the nominal closed-loop performance (through $\overline{\boldsymbol{x}}_{\text {nom }}, y_{c}$ ), the modeling error (through $\boldsymbol{W}^{T} \boldsymbol{\phi}(\boldsymbol{\eta})$ ), and the degree of NN adaptation to the modeling error (through $\boldsymbol{e}_{m}, \boldsymbol{z}$ ). The following technical assumption requires that $u_{h}^{*}$ satisfies a linear growth condition on the domain of interest $\Omega_{e_{m}}, \Omega_{z}$. The set $\Omega_{e_{m}}$ is defined in Eq. (39), and $\Omega_{z}$ represents the domain of interest for $z$ in Eq. (26). In case of input saturation, the assumption implies that the deficient control caused by control limit satisfies the linear growth condition.
Assumption 6: The control hedging signal $u_{h}^{*}$ is bounded as follows:

$$
\left|u_{h}^{*}\right| \leq \mu_{1}\left\|\boldsymbol{e}_{m}\right\|+\mu_{2}\|z\|+\mu_{4} \quad \text { for } \quad \boldsymbol{e}_{m} \in \Omega_{e_{m}}, \quad z \in \Omega_{z}
$$

Compared to $u_{h}^{*}$ in Eq. (45), $u_{h}$ in Eq. (37) can be expressed as

$$
\begin{aligned}
u_{h}= & u_{h}^{*}+D_{r}^{-1}\left(v_{\mathrm{ad}}^{*}-v_{\mathrm{ad}}\right)+\left(\hat{g}\left(u_{\mathrm{lc}}+D_{r}^{-1}\left(v_{\mathrm{dc}}-v_{\mathrm{ad}}^{*}\right)\right)\right. \\
& \left.-\hat{g}\left(u_{\mathrm{lc}}+D_{r}^{-1}\left(v_{\mathrm{dc}}-v_{\mathrm{ad}}\right)\right)\right)
\end{aligned}
$$

Using Eq. (47), with assumption $5, u_{h}$ is bounded by

$$
\begin{aligned}
& \left|u_{h}\right| \leq \mu_{1}\left\|\boldsymbol{e}_{m}\right\|+\mu_{2}\|z\|+\mu_{3}\|\tilde{\boldsymbol{W}}\|+\mu_{4} \\
& \qquad \text { for } \quad \boldsymbol{e}_{m} \in \Omega_{e_{m}}, \quad z \in \Omega_{z}
\end{aligned}
$$

where $\mu_{3}=(1+L)\|\phi\|$.

We will show via Lyapunov's direct method that the signals $\boldsymbol{e}_{m}$ in Eq. (39), $\boldsymbol{z}$ in Eq. (26), $\boldsymbol{z}_{f}$ in Eq. (29), and NN weight errors $\tilde{\boldsymbol{W}}$ are bounded. With that objective in mind, we define the error vector $\zeta^{T} \triangleq\left[\begin{array}{llll}\boldsymbol{e}_{m}^{T} & \boldsymbol{z}^{T} & \boldsymbol{z}_{f}^{T} & \tilde{\boldsymbol{W}}^{T}\end{array}\right]$, which belongs to the convex compact set $\mathcal{B}_{R} \triangleq\{\zeta \mid\|\zeta\| \leq R, R>0\} \subseteq \Omega_{e_{m}} \times \Omega_{z} \times \Omega_{z_{f}} \times \Omega_{\tilde{W}}$ such that for every $\zeta \in \mathcal{B}_{R}$, the NN approximation implied in Eq. (17), with $\Delta$ defined in Eq. (42), is valid. Consider the following Lyapunov function candidate:

$$
L(\zeta)=\zeta^{T} T \zeta=\frac{1}{2} \boldsymbol{e}_{m}^{T} P_{n} \boldsymbol{e}_{m}+\frac{1}{2} z^{T} P z+\frac{1}{2} z_{f} P_{f} z_{f}+\frac{1}{2} \operatorname{tr}\left\{\tilde{\boldsymbol{W}}^{T} \Gamma_{W}^{-1} \tilde{\boldsymbol{W}}\right\}
$$

where $P_{n}, P, P_{f}>0$ are solutions of Eqs. (40), (28), and (30) respectively and $T$ is defined to be

$$
T=\frac{1}{2}\left[\begin{array}{cccc}
P_{n} & 0 & 0 & 0 \\
0 & P & 0 & 0 \\
0 & 0 & P_{f} & 0 \\
0 & 0 & 0 & \Gamma_{W}^{-1}
\end{array}\right]
$$

Introduce $T_{m}, T_{M}$, which are minimal and maximal eigenvalues of $T$, respectively. Then $T_{m}\|\zeta\|^{2} \leq L(\zeta) \leq T_{M}\|\zeta\|^{2}$. Let $\alpha=$ $\sqrt{ }\left(T_{m} / T_{M}\right) R, \mathcal{B}_{\alpha}=\left\{\zeta \in \mathcal{B}_{R} \mid\|\zeta\| \leq \alpha\right\}$.

Theorem 4: Suppose $\zeta(0) \in \mathcal{B}_{\alpha}$ and

$$
R>\sqrt{T_{M} / T_{m}} C \geq C
$$

where $C$ is defined later in Eq. (A9). Subject to assumptions 1-6, the control law $u=u_{\mathrm{lc}}+D_{r}^{-1}\left(v_{\mathrm{dc}}-v_{\mathrm{ad}}\right)$ with plant model dynamics in Eq. (36) guarantees that the signal $\zeta$ is uniformly ultimately bounded with the bound $\sqrt{ }\left(T_{M} / T_{m}\right) C$, provided the following conditions hold:

$$
\begin{gathered}
\gamma_{1}>\gamma_{4}, \quad \lambda_{\min }(Q)>2 \epsilon\left\|P \boldsymbol{B}_{\mathrm{cl}}\right\|+\frac{4 \gamma_{2}^{2}}{\gamma_{1}} \\
\lambda_{\min }\left(Q_{f}\right)>\left\|P_{f} \boldsymbol{B}_{f}\right\|\|\phi\|, \quad \sigma>1+\frac{\gamma_{3}^{2}}{\gamma_{1}}+\frac{\alpha^{2}\left\|P \boldsymbol{B}_{\mathrm{cl}}\right\|^{2}}{\lambda_{\min }(Q)}
\end{gathered}
$$

where $\gamma_{1}=\frac{1}{2} \lambda_{\min }\left(Q_{n}\right)-\mu_{1}\left\|P_{n} \boldsymbol{G}\right\|, \gamma_{2}=\left\|P_{n} \boldsymbol{G}_{c} \boldsymbol{C}_{\tilde{y}}\right\|+\mu_{2}\left\|P_{n} \boldsymbol{G}\right\|$, $\gamma_{3}=\mu_{3}\left\|P_{n} \boldsymbol{G}\right\|, \gamma_{4}=\mu_{4}\left\|P_{n} \boldsymbol{G}\right\|$.

Proof: See Appendix.

The uniform ultimate boundedness of $\boldsymbol{e}_{m}$ and $z$ guarantees, using Eqs. (8) and (26), that the tracking error is bounded; $\left|y_{c}-y\right| \leq$ $\left|y_{c}-y_{\text {nom }}\right|+\left|y_{\text {nom }}-y_{m}\right|+|\tilde{y}| \leq\left|y_{c}-y_{\text {nom }}\right|+\left\|\boldsymbol{C}_{m}\right\|\left\|\boldsymbol{e}_{m}\right\|+\left\|\boldsymbol{C}_{e}\right\|$ $\left\|z_{e}\right\|$, where $\left|y_{c}-y_{\text {nom }}\right|$ represents the nominal bound for tracking error in Eq. (8). 
Fig. 4 Three-disk torsional pendulum system. (Data available online at http://www.ecpsystems. com/controls_torplant.htm, cited 16 January 2004.)

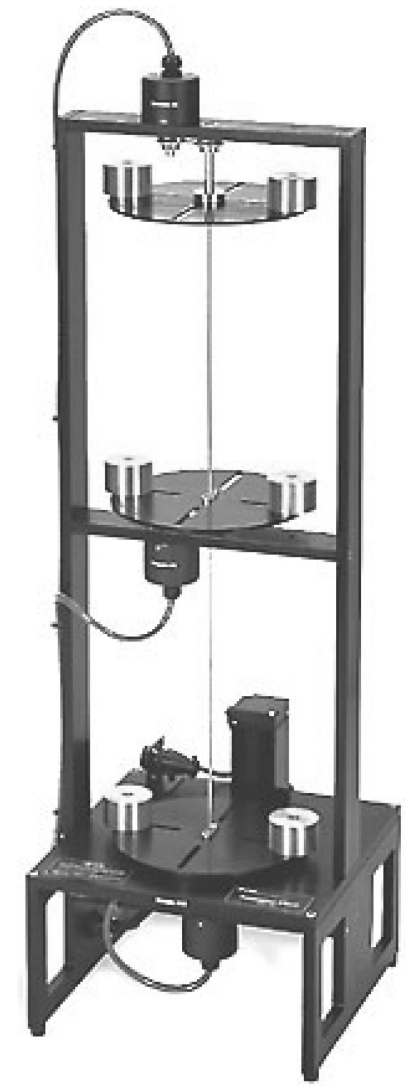

Remark 2: The assumption that $\zeta(0) \in \mathcal{B}_{\alpha}$ implies that the control system initially belongs to the domain where stabilization is possible because when $\zeta \in \mathcal{B}$ and $\|\zeta\|>C, \dot{L}<0$. If the system initially belongs to the region in which it cannot be stabilized, the control hedging signal $u_{h}$ goes unbounded, resulting in $\boldsymbol{e}_{m} \notin \Omega_{e_{m}}$ after some time.

Remark 3: The assumption that $R>\sqrt{ }\left(T_{M} / T_{m}\right) C \geq C$ implies both an upper and a lower bound for the adaptation gain. Define $\bar{\gamma} \triangleq \max \Gamma_{W}, \quad \bar{\gamma} \triangleq \min \Gamma_{W}, \quad \bar{\lambda}=\max \left(\lambda_{\max } P_{n}, \lambda_{\max } P, \lambda_{\max } P_{f}\right)$, $\underline{\lambda}=\min \left(\lambda_{\min } P_{n}, \lambda_{\min } P, \lambda_{\min } P_{f}\right)$, where $\lambda(\cdot)$ denotes a eigenvalue. If the adaptation gain is large such that $\gamma>1 / \bar{\lambda}$, Eq. (52) implies an upper bound $\bar{\gamma}<R^{2} / C^{2} \bar{\lambda}$ for the adaptation gain. Likewise, for $\bar{\gamma}<1 / \bar{\lambda}$, Eq. (52) leads to a lower bound $\gamma>C^{2} / R^{2} \underline{\lambda}$ for the adaptation gain.

Remark 4: The closed-loop system when the NN adaptation is exact, that is, $v_{\mathrm{ad}}=v_{\mathrm{ad}}^{*} \Rightarrow u_{h}=u_{h}^{*}$, is defined as a nonadaptive subsystem in Ref. 17. The performance of this system represents the best performance that can be achieved with $\mathrm{CH}$ together with the NN-based adaptive element. The uniform ultimate bounded region, in this case, further shrinks because all of the constants related to $\tilde{\boldsymbol{W}}$ vanishes from $C$ in Eq. (A9).

\section{Design and Experimental Results with a Three-Disk Torsional Pendulum System}

Figure 4 depicts a torsional pendulum system that is made up of three disks connected by a flexible shaft.* Only the bottom disk is actuated by a brushless dc servomotor. The equations of motion for the system are as follows:

$$
\begin{gathered}
J_{1} \ddot{\theta}_{1}+B \dot{\theta}_{1}+K\left(\theta_{1}-\theta_{2}\right)+f_{c_{1}}\left(\dot{\theta}_{1}, \theta_{1}, \theta_{2}\right)=K_{d} V_{d} \\
J_{2} \ddot{\theta}_{2}+B \dot{\theta}_{2}-K \theta_{1}+2 K \theta_{2}-K \theta_{3}+f_{c_{2}}\left(\dot{\theta}_{2}, \theta_{1}, \theta_{2}, \theta_{3}\right)=0 \\
J_{3} \ddot{\theta}_{3}+B \dot{\theta}_{3}-K\left(\theta_{2}-\theta_{3}\right)+f_{c_{3}}\left(\dot{\theta}_{3}, \theta_{2}, \theta_{3}\right)=K_{v t} u
\end{gathered}
$$

where $J_{i}=0.103 \mathrm{~kg} \cdot \mathrm{m}^{2} ; i=1,2,3$ are the moments of inertia; $B=0.0018 \mathrm{~kg} \cdot \mathrm{m} / \mathrm{s}$ is the viscous damping coefficient; $K=$ $2.2625 \mathrm{~kg} \cdot \mathrm{m}^{2} / \mathrm{s}^{2}$ is the spring constant; $K_{d}=0.05 \mathrm{~N} \cdot \mathrm{m} / \mathrm{V}$ is the gain from disturbance voltage to torque; $K_{\mathrm{vt}}=0.42 \mathrm{~N} \cdot \mathrm{m} / \mathrm{V}$ is the gain from control voltage to torque; and $f_{c i}$ represents nonlinearities, such as coulomb friction. The control input $u$ is the voltage applied to the control motor, and the disturbance input $V_{d}$ is the voltage applied to the disturbance drive. The regulated output variable is the angular displacement of the bottom disk $\theta_{3}$, constituting a collocated control problem. The output has relative degree two if the dynamics of the dc motor are treated as lying outside the bandwidth of the design. With $f_{c_{i}}=0$, the transfer function from applied voltage to the regulated output is given by

$$
\frac{y}{u}=\frac{K_{a}\left(s^{2}+2 \zeta_{z_{1}} \omega_{z_{1}} s+\omega_{z_{1}}^{2}\right)\left(s^{2}+2 \zeta_{z_{2}} \omega_{z_{2}} s+\omega_{z_{2}}^{2}\right)}{s(s+c)\left(s^{2}+2 \zeta_{p_{1}} \omega_{p_{1}} s+\omega_{p_{1}}^{2}\right)\left(s^{2}+2 \zeta_{p_{2}} \omega_{p_{2}} s+\omega_{p_{2}}^{2}\right)}
$$

The parameters are $K_{a}=40.46, \zeta_{z_{1}}=0.009, \omega_{z_{1}}=9.87, \zeta_{z_{2}}=$ $0.0035, \omega_{z_{2}}=25.8, c=0.1786, \zeta_{p_{1}}=0.00559, \omega_{p_{1}}=16(\mathrm{rad} / \mathrm{s})$, $\zeta_{p_{2}}=0.00323$, and $\omega_{p_{2}}=27.7(\mathrm{rad} / \mathrm{s})$. The eigenvalues associated with the zero dynamics are $-0.089 \pm 15.97 i$ and $-0.893 \pm 27.66 i$. Therefore the system is globally exponentially minimum phase. Thus the assumptions 1 and 2 are easily verified.

To emphasize the presence of unmodeled dynamics, the following low-frequency model for the plant dynamics that does not include the flexible modes is assumed:

$$
y_{m} / u=K_{n} / s(s+c)
$$

where $K_{n}=13.49$ is determined so that low-frequency gain of the plant model matches that of the sixth-order plant. Figure 5 compares the frequency response of the assumed plant model with that of the higher-fidelity model. The agreement is quite good at low frequencies but differs significantly at high frequencies because of the unmodeled flexible modes. Comparison of Eq. (53) with Eq. (55) leads to the following modelling error:

$$
\begin{aligned}
\Delta= & -\left(B / J_{3}\right) \dot{\theta}_{3}+\left(K / J_{3}\right)\left(\theta_{2}-\theta_{3}\right)-\left(1 / J_{3}\right) f_{c_{3}}\left(\dot{\theta}_{3}, \theta_{2}, \theta_{3}\right) \\
& +\left(K_{\mathrm{vt}} / J_{3}\right) u+c \dot{y}_{m}-K_{n} u
\end{aligned}
$$

By simple manipulation, we can ensure that assumption 4 is satisfied if $K_{n}-K_{\mathrm{vt}} / J_{3} \neq D_{r}$.

The linear controller is designed as a lead compensator, which results in a dominant mode at $\omega_{n}=3 \mathrm{rad} / \mathrm{s}$ and $\zeta=0.8$ for the nominal
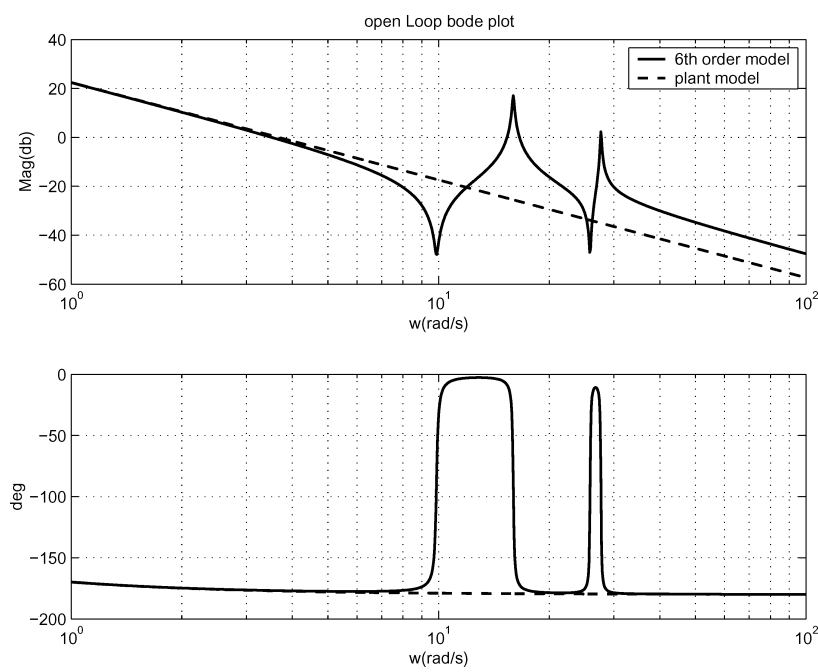

Fig. 5 Open-loop Bode plot for the sixth-order model and the plant model.

\footnotetext{
*Data available online at http://www.ecpsystems.com/controls_torplant. htm [cited 16 January 2004].
} 
system design. This results in

$$
u_{\mathrm{lc}}=K_{1}\left[\left(s+b_{1}\right) /\left(s+a_{1}\right)\right]\left(y_{c}-y\right)
$$

where $K_{1}=0.67, a_{1}=4.8$, and $b_{1}=0.1786$. The error compensator and the SPR filter are designed as

$$
\left[\begin{array}{c}
v_{\mathrm{dc}}(s) \\
\tilde{y}_{\mathrm{ad}}(s)
\end{array}\right]=\frac{1}{s+42.6}\left[\begin{array}{c}
427(s+3.44) \\
40(s+5)
\end{array}\right] \tilde{y}, \quad T^{-1}(s)=\frac{1}{s+1}
$$

A radial basis function $\mathrm{NN}$ of 78 neurons is used to approximate for the system uncertainty. Its input is constructed as in Eq. (18) using two delayed values of control signal $u$ and five delayed values of output $y$ with a delay $d=0.1 \mathrm{~s}$. That is, $\bar{u}_{d}^{T}(t)=$ $[u(t) u(t-d) u(t-2 d)]^{T}, \bar{y}_{d}^{T}(t)=[y(t) y(t-d) \cdots y(t-5 d)]^{T}$. To circumvent a fixed-point iteration in the real-time environment, the control signal is further delayed before it is used as the network input. In simulation, this was compared to obtaining a fixed-point solution, and the results were not distinguishable.

Radial basis functions are employed in the $\mathrm{NN}$ :

$$
\phi_{k}(\boldsymbol{\eta})=e^{-\left\|\eta-\eta_{c_{k}}\right\|^{2} / 2 \sigma_{k}}, \quad \sigma_{k}=\sqrt{5}, \quad k=1, \ldots, 78
$$

The centers $\boldsymbol{\eta}_{c_{k}}$ are randomly selected over a grid of possible values for the vector $\boldsymbol{\eta}$. All of the NN inputs are normalized using an estimate for their maximum values. Adaptation gains are chosen as $\Gamma_{W}=100$ and $\sigma=0.5$.

Figure 6 compares the response of the regulated output with and without the adaptive element for a square wave command of $20 \mathrm{deg}$. The response with the controller in Eq. (57), and without the adaptive element, is shown as dashed lines. The reference command and the response with the adaptive element are shown as solid lines. The absence of the flexible modes in the response with the adaptive element shows that the augmented controller provides adaptation to the unmodeled flexible modes. Furthermore, the output response without the adaptive element resulted in a large steady-state error. This is caused by stiction that is also unmodeled in the nominal controller design. The output response with the adaptive element reveals that the effect of stiction on the output response is removed.

An alternative approach to removing steady-state error is to add integral action in the linear controller. A lead/lag controller was design with integral action (low-frequency pole of the compensator at zero) to provide approximately the same transient response when applied to linear model in Eq. (55). This design increased the loop crossover frequency from 1.8 to $2.5 \mathrm{rad} / \mathrm{s}$ and reduced the phase margin from 70 to $55.6 \mathrm{deg}$. However, because of the nonlinear stiction characteristic of the actuator, this resulted in a nonlinear response to a step command that ultimately staircases to a nearzero steady-state error. In essence, the integrator repeatedly winds

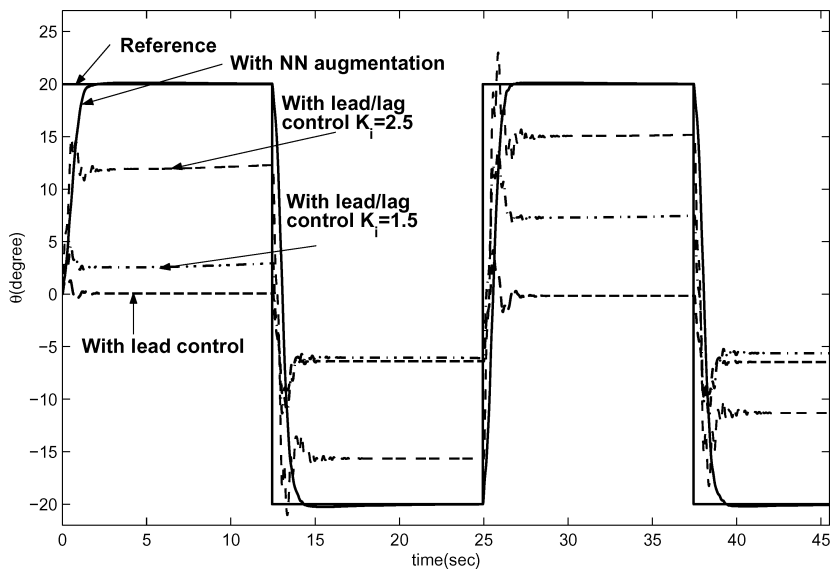

Fig. 6 Comparison of the output responses of the true plant with and without the adaptive element. up until it overcomes the stiction. The experimental results for a response to a square wave command are depicted in Fig. 6 for the design value of the integral gain $\left(K_{i}=1.5\right)$ and a higher value of integral gain $\left(K_{i}=2.5\right)$. The staircase is not seen in this response because the command is not held constant for a sufficient duration. The increased destabilizing effect that this controller has on the flexible modes can also be seen. In contrast the response with the adaptive element (and without integral control) is nearly identical to the ideal model response (the response of the plant model regulated by the lead controller), does not exhibit a nonlinear characteristic, and completely eliminates the effect of the unmodeled modes.

To demonstrate both good tracking and attenuation of disturbances by the augmented controller, a set of reference commands and disturbances is combined in an experiment with the results shown in Fig. 7. For this case, the bandwidth of the linear controller is increased to $\omega_{n}=5 \mathrm{rad} / \mathrm{s}$. A disturbance is applied as an external torque to the system using a friction drive motor, which is attached to the rim of the top disk. This introduces unmodeled dynamics associated with the disturbance process. Hence, the disturbance is not matched because it is noncollocated with the control. The voltage applied to the rim drive motor is

$V_{d}(t)= \begin{cases}0.7 \sin 15.7 t & (0 \leq t \leq 22) \\ 0.4(\sin t+\sin 3 t+\sin 15.7 t+\sin 27.7 t) & (22 \leq t \leq 45)\end{cases}$
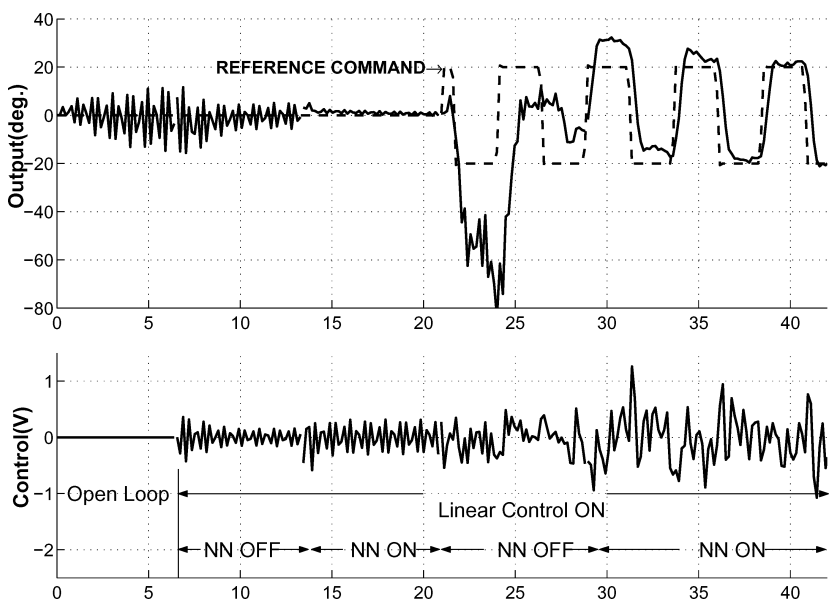

Fig. 7 Experimental results with and without the adaptive element.

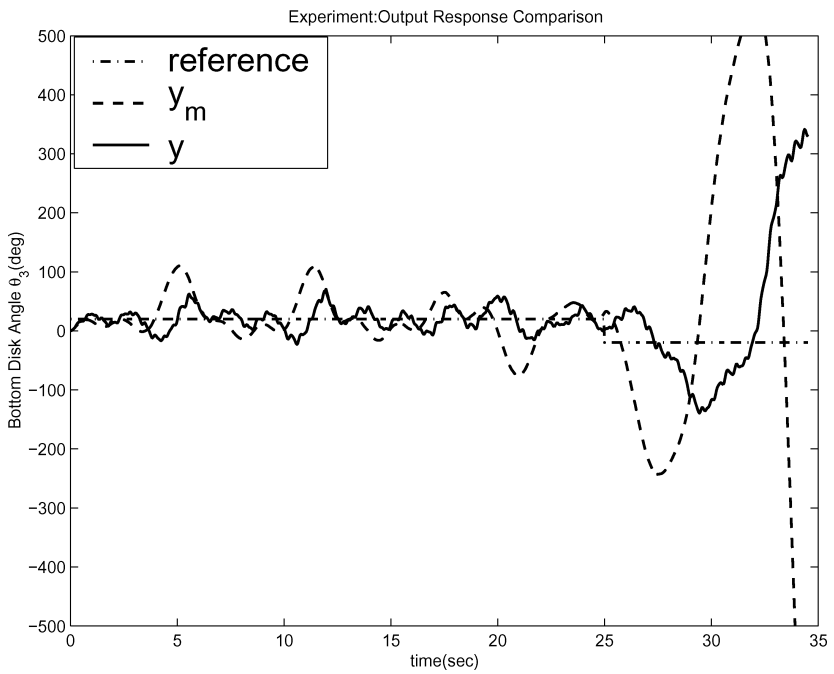

Fig. 8 Responses of plant model $y_{m}$ and the output $y$ without $\mathrm{CH}$. 
where $V_{d}(t)$ is the voltage applied to the disturbance drive motor. With the disturbance in Eq. (60), it is straightforward to check that the disturbance dynamics have eigenvalues that are distinct from those of the plant dynamics in Eq. (54). The augmented dynamics consisting of plant dynamics and disturbance dynamics are observable by theorem 3 . Thus assumption 3 is satisfied.

In Fig. 7 the upper traces show the output response (angular position of the lower disk) while the lower trace shows the control voltage. The reference command is zero for the first $22 \mathrm{~s}$ and is a $0.2-\mathrm{Hz}$ square wave of magnitude $20 \mathrm{deg}$ for the remaining $23 \mathrm{~s}$.

The results during the first $7 \mathrm{~s}$ show the open-loop response. The linear controller is activated at $t=7 \mathrm{~s}$. and is able to noticeably reduce the output response to the disturbance. At $t=14 \mathrm{~s}$, the adaptive element is turned on and is able to essentially cancel the effect of the disturbance in the output response. At $t=22 \mathrm{~s}$ the adaptive element is turned off, and the square wave is applied as the command to test tracking performance. At the same time, the disturbance signal $V_{d}(t)$ is modified as defined in Eq. (60). Under these conditions, the linear controller is clearly not able to track the reference command, and the response deviates wildly. At $t=29 \mathrm{~s}$, the adaptive element is turned on again and is quickly able to begin tracking the command with increasing fidelity as the $\mathrm{NN}$ adapts.

In practice the applied control voltage must be limited because excessive angular displacement between two disks can damage the flexible shaft connecting them. This amounts to introducing a form of control saturation, but the $\mathrm{CH}$ method can be employed to allow the adaptive process to continue during saturation. In the next

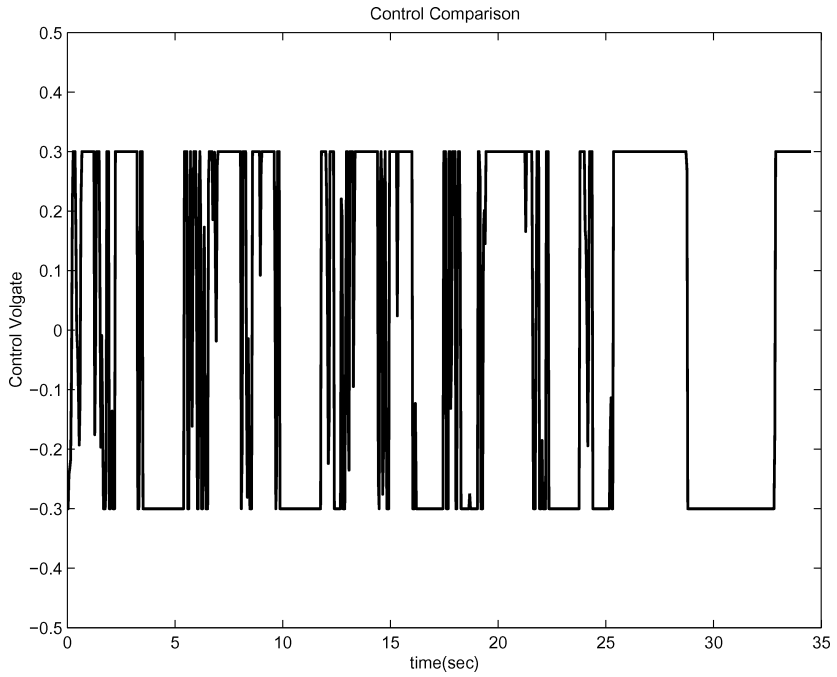

Fig. 9 Control voltage with limit $0.3 \mathrm{~V}$ imposed without $\mathrm{CH}$.

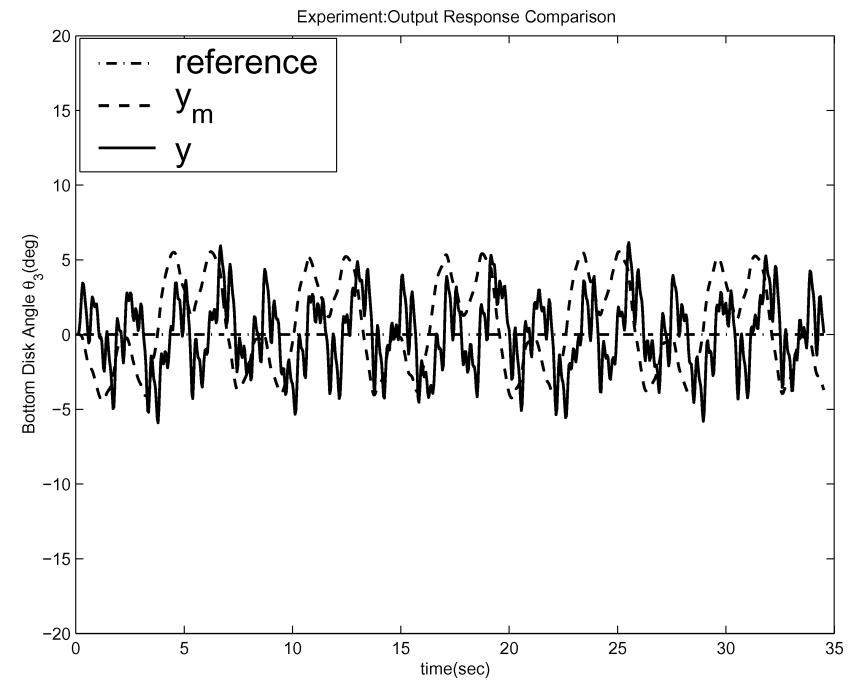

Fig. 10 Responses of plant model $y_{m}$ and the output $y$ with $\mathrm{CH}$. experiment, a control limit of $0.3 \mathrm{~V}$ is introduced. Because it is implemented in software, $\delta$ in Eq. (33) equals $\hat{\delta}$ in Eq. (35). The input saturation globally satisfies the assumption 5 with Lipschitz constant $L=1$. It is also straightforward to check that the control deficiency because of input saturation satisfies assumption 6 .

The reference command is set to zero, and the disturbance is constructed as $V_{d}(t)=0.5(\sin t+\sin 3 t+\sin 12 t+\sin 15.7 t+$ $\sin 27.7 t$ ). Because the control voltage limit is set to a value that does not permit cancellation of the disturbance, the disturbance is expected to be only partially attenuated. The main focus in this experiment is to show that the $\mathrm{CH}$ technique ensures correct adaptation while the actuator is in saturation. Figure 8 shows the response when the adaptive element is active, but without $\mathrm{CH}$. Incorrect adaptation is evidenced by a growing response and the difference between the actual plant $(y)$ and the plant model $\left(y_{m}\right)$ under the continual input saturation as shown in Fig. 9. The NN weights also diverge because of control saturation. Figure 10 shows the response for the same controller, but with $\mathrm{CH}$ active. Although the disturbance is not completely cancelled, the output tracks the plant model response reasonably well. This illustrates that correct adaptation is achieved with $\mathrm{CH}$, even when there is a significant amount of control saturation as in Fig. 11. The NN weight histories (not shown) were also bounded.

The control architecture was also tested on the noncollocated control problem in which the angular position of the middle disk was chosen as the regulated output. In this case, the regulated output has relative degree four. The plant model is derived assuming the shaft that connects the middle and top disk is rigid. A linear quadratic

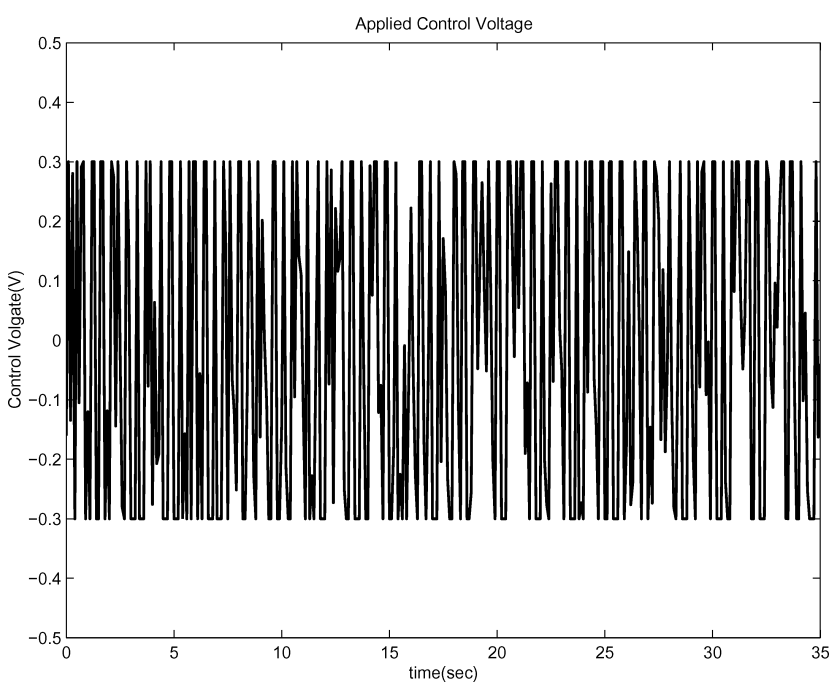

Fig. 11 Control voltage and control hedging signal with limit $0.3 \mathrm{~V}$ imposed with $\mathrm{CH}$.

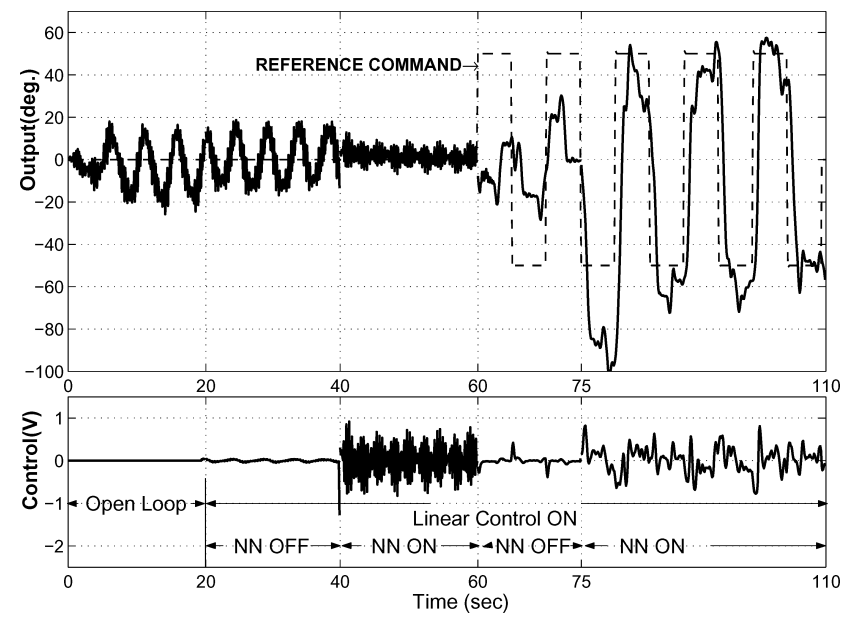

Fig. 12 Experimental results on the middle disk control. 
Gaussian (LQG) controller is designed as a linear controller. To improve observability of the overall system, the moment of inertia of the bottom disk is increased by increasing the mass mounted on the bottom disk. The LQG controller is designed without taking into account the increase in inertia. The disturbance is applied in the same manner as in the earlier experiment just described. The experiment is carried out in the same manner as collocated control. Representative output responses are shown in Fig. 12. It shows that the LQG controller performs very poorly because of the modeling error, even though it showed good tracking and reasonable disturbance attenuation in simulation with the plant model. With the augmented controller, the responses are similar to those in Fig. 7; however, NN adaptation is slower compared to that observed in the collocation control case. When a voltage limit is applied, results similar to those illustrated in Figs. 8-11 were obtained.

\section{Conclusions}

This paper describes an approach for augmenting a linear controller design with an adaptive element. The linear controller can either be an existing controller or it can be a controller that is explicitly designed as a part of the adaptive controller design itself. The key properties of the design are that only output variables are used, and it is adaptive to both parametric errors and unmodeled/unmatched dynamics and disturbances. The main assumptions are that the relative degree of the regulated output is known, the plant is observable, and the closed-loop system has stable zero dynamics. Experimental results obtained using a three-disk torsional pendulum laboratory model illustrate the effectiveness of the design approach, including the incorporation of a novel approach that permits adaptation during periods of control saturation.

\section{Appendix: Proofs}

Proof of Theorem 3: By the Popov-Belevitch-Hautus (PBH) test $^{28}$, the linear system defined with matrices in Eq. (32) is observable if

$$
\operatorname{rank}\left[\begin{array}{cc}
\boldsymbol{C}_{p} & {\left[\begin{array}{c}
\boldsymbol{D}_{p} \\
-\boldsymbol{B}_{p}
\end{array}\right] \boldsymbol{C}_{d}} \\
s \boldsymbol{I}_{n_{p}}-\boldsymbol{A}_{p} & \boldsymbol{I}_{n_{d}}-\boldsymbol{A}_{d}
\end{array}\right]=n, \quad \forall s \in C
$$

Because of the zero block in (A1), when $\boldsymbol{A}_{p}$ and $\boldsymbol{A}_{d}$ have no common eigenvalues, observability of Eq. (32) is ensured if $\left(\boldsymbol{A}_{p}, \boldsymbol{C}_{p}\right)$ is observable and $\left(\boldsymbol{A}_{d}, \overline{\boldsymbol{C}}\right)$ is observable, where

$$
\overline{\boldsymbol{C}}=\left[\begin{array}{c}
\boldsymbol{D}_{p} \\
-\boldsymbol{B}_{p}
\end{array}\right] \boldsymbol{C}_{d}
$$

It remains to be shown that observability of $\left(\boldsymbol{A}_{d}, \overline{\boldsymbol{C}}\right)$ is equivalent to observability of $\left(\boldsymbol{A}_{d}, \boldsymbol{C}_{d}\right)$. From the PBH eigenvector test, ${ }^{28}\left(\boldsymbol{A}_{d}, \overline{\boldsymbol{C}}\right)$ is not observable if there exists a vector $\boldsymbol{q} \neq 0$ such that $\boldsymbol{A}_{d} \boldsymbol{q}=\lambda \boldsymbol{q}$ and $\overline{\boldsymbol{C}} \boldsymbol{q}=0$. By assumption 3 in theorem $3, \overline{\boldsymbol{C}} \boldsymbol{q}=0$ if $\boldsymbol{C}_{d} \boldsymbol{q}=0$.

Proof of Corollary 1: If $\boldsymbol{A}_{p}$ and $\boldsymbol{A}_{d}$ do not share a common eigenvalue, then from theorem 3 it follows that $(\boldsymbol{A}, \boldsymbol{C})$ in Eq. (32) is observable. Suppose $\boldsymbol{A}_{p}$ and $\boldsymbol{A}_{d}$ do share a common eigenvalue at $s=\lambda$. Then by PBH eigenvector test $(\boldsymbol{A}, \boldsymbol{C})$ is not observable if there exist $\overline{\boldsymbol{q}} \neq 0$ such that

$$
\left[\begin{array}{cc}
\boldsymbol{C}_{p} & {\left[\begin{array}{c}
\boldsymbol{D}_{p} \\
-\boldsymbol{B}_{p}
\end{array}\right] \boldsymbol{C}_{d}} \\
\lambda \boldsymbol{I}_{n_{p}}-\boldsymbol{A}_{p} & \boldsymbol{I}_{n_{d}}-\boldsymbol{A}_{d}
\end{array}\right] \overline{\boldsymbol{q}}=0
$$

Let $\boldsymbol{q}_{2}$ be an eigenvector of $\boldsymbol{A}_{d}$ corresponding to $\lambda$, then there exists a $\boldsymbol{q}_{1}$ such that

$$
\left[\begin{array}{c}
\boldsymbol{C}_{p} \\
\lambda \boldsymbol{I}_{n_{p}}-\boldsymbol{A}_{p}
\end{array}\right] \boldsymbol{q}_{1}+\overline{\boldsymbol{C}} \boldsymbol{q}_{2}=0
$$

if $\overline{\boldsymbol{C}} \boldsymbol{q}_{2}$ lies in the column space of $\left[\begin{array}{lll}\boldsymbol{C}_{p}^{T} & \lambda \boldsymbol{I}_{n_{p}}-\boldsymbol{A}_{p}^{T}\end{array}\right]$.
Proof of Theorem 4: Consider the Lyapunov function candidate $L$ in Eq. (50). With $\mathrm{NN}$ update rule in Eq. (31) and using $\boldsymbol{z}^{T} P \boldsymbol{B}_{\mathrm{cl}}=\boldsymbol{z}^{T} \boldsymbol{C}_{\mathrm{cl}}^{T}=\tilde{y}_{\mathrm{ad}}$ from Eq. (28), together with the dynamics described in Eqs. (39), (26), and (29), the time derivative $\dot{L}$ is described by

$$
\begin{aligned}
\dot{L}= & -\frac{1}{2} \boldsymbol{e}_{m}{ }^{T} Q_{n} \boldsymbol{e}_{m}+\boldsymbol{e}_{m}^{T} P_{n}\left[-\boldsymbol{G}_{c} \tilde{y}+\boldsymbol{G} u_{h}\right] \\
& -\frac{1}{2} z^{T} Q z+z^{T} P \boldsymbol{B}_{\mathrm{cl}}\left(\theta-\varepsilon_{f}\right) \\
& -\frac{1}{2} \boldsymbol{z}_{f}^{T} Q_{f} \boldsymbol{z}_{f}+\boldsymbol{z}_{f}^{T} P_{f} \boldsymbol{B}_{f} \boldsymbol{\phi}-\sigma \operatorname{tr}\left\{\tilde{\boldsymbol{W}}^{T}(\tilde{\boldsymbol{W}}+\boldsymbol{W})\right\}
\end{aligned}
$$

where $Q_{n}, Q, Q_{f}$ are defined in Eqs. (40), (28), and (30), respectively. Assuming that the filter $T^{-1}(s)$ is scaled so that its maximum gain is unity, the filtered error $\varepsilon_{f}$ can be bounded as

$$
\left|\varepsilon_{f}\right| \leq \epsilon
$$

With this bound, the time derivative $\dot{L}$ is upper bounded by

$$
\begin{aligned}
\dot{L} \leq & -\frac{1}{2} q_{n}\left\|\boldsymbol{e}_{m}\right\|+\left\|\boldsymbol{e}_{m}\right\|\|\boldsymbol{z}\|\left\|P_{n} \boldsymbol{G}_{c} \boldsymbol{C}_{\tilde{y}}\right\|+\left\|\boldsymbol{e}_{m}\right\|\left\|P_{n} \boldsymbol{G}\right\|\left|u_{h}\right| \\
& -\frac{1}{2} q\|z\|+\|z\| \| P \boldsymbol{B}_{\mathrm{cl}}[|\theta|+\epsilon] \\
& -\frac{1}{2} q_{f}\left\|z_{f}\right\|+\left\|z_{f}\right\|\left\|P_{f} \boldsymbol{B}_{f} \phi\right\|-\sigma \operatorname{tr}\left\{\tilde{\boldsymbol{W}}^{T}(\tilde{\boldsymbol{W}}+\boldsymbol{W})\right\}
\end{aligned}
$$

where $q_{n}=\lambda_{\min }\left(Q_{n}\right), q=\lambda_{\min }(Q)$, and $q_{f}=\lambda_{\min }\left(Q_{f}\right)$. Using the inequality in Eq. (49), $\dot{L}$ is arranged as

$$
\begin{aligned}
\dot{L} & \leq-\gamma_{1}\left\|\boldsymbol{e}_{m}\right\|^{2}+\gamma_{2}\left\|\boldsymbol{e}_{m}\right\|\|z\|+\gamma_{3}\left\|\boldsymbol{e}_{m}\right\|\|\tilde{\boldsymbol{W}}\|+\gamma_{4}\left\|\boldsymbol{e}_{m}\right\| \\
& -\frac{1}{2} q\left\|z^{2}\right\|+\beta_{1}\|z\|\|\tilde{\boldsymbol{W}}\|+\beta_{2}\|\boldsymbol{z}\| \\
& -\frac{1}{2} q_{f}\left\|\boldsymbol{z}_{f}\right\|^{2}+\beta_{3}\left\|z_{f}\right\|-\sigma\|\tilde{\boldsymbol{W}}\|_{F}^{2}+\sigma\|\tilde{\boldsymbol{W}}\| W^{*}
\end{aligned}
$$

where

$$
\begin{array}{ccc}
\gamma_{1}=\frac{1}{2} q_{n}-\mu_{1}\left\|P_{n} \boldsymbol{G}\right\|, & \gamma_{2}=\left\|P_{n} \boldsymbol{G}_{c} \boldsymbol{C}_{\tilde{y}}\right\|+\mu_{2}\left\|P_{n} \boldsymbol{G}\right\| \\
\gamma_{3}=\mu_{3}\left\|P_{n} \boldsymbol{G}\right\|, & \gamma_{4}=\mu_{4}\left\|P_{n} \boldsymbol{G}\right\|, & \beta_{1}=\alpha\left\|P \boldsymbol{B}_{\mathrm{cl}}\right\| \\
\beta_{2}=\epsilon\left\|P \boldsymbol{B}_{\mathrm{cl}}\right\|, & \beta_{3}=\left\|P_{f} \boldsymbol{B}_{f} \phi\right\|, & \|\boldsymbol{W}\| \leq W^{*}
\end{array}
$$

Upon completion of square, $\dot{L}$ can be regrouped as

$$
\begin{aligned}
\dot{L} \leq & -\frac{\gamma_{1}}{2}\left\|\boldsymbol{e}_{m}\right\|^{2}-\frac{\gamma_{1}}{4}\left[\left\|\boldsymbol{e}_{m}\right\|-\frac{2 \gamma_{2}}{\gamma_{1}}\|\boldsymbol{z}\|\right]^{2}+\frac{\gamma_{2}^{2}}{\gamma_{1}}\|\boldsymbol{z}\|^{2} \\
& -\frac{\gamma_{1}}{4}\left[\left\|\boldsymbol{e}_{m}\right\|-\frac{2 \gamma_{3}}{\gamma_{1}}\|\tilde{\boldsymbol{W}}\|_{F}\right]^{2}+\frac{\gamma_{3}^{2}}{\gamma_{1}}\|\tilde{\boldsymbol{W}}\|_{F}+\gamma_{4}\left\|\boldsymbol{e}_{m}\right\| \\
& -\frac{1}{4} q\|\boldsymbol{z}\|^{2}-\frac{q}{4}\left[\|\boldsymbol{z}\|-\frac{2 \beta_{1}}{q}\|\tilde{\boldsymbol{W}}\|_{F}\right]^{2}+\frac{\beta_{1}^{2}}{q}\|\tilde{\boldsymbol{W}}\|_{F}^{2}+\beta_{2}\|\boldsymbol{z}\| \\
& -\frac{1}{2} q_{f}\left\|\boldsymbol{z}_{f}\right\|^{2}+\beta_{3}\left\|\boldsymbol{z}_{f}\right\|-\sigma\|\tilde{\boldsymbol{W}}\|_{F}^{2}+\sigma\|\tilde{\boldsymbol{W}}\|_{F} W^{*}
\end{aligned}
$$

The terms that involve products of variables and are linear in the variables are upper bounded as follows:

$$
\begin{gathered}
\gamma_{4}\left\|\boldsymbol{e}_{m}\right\| \leq\left(\gamma_{4} / 2\right)\left[\left\|\boldsymbol{e}_{m}\right\|^{2}+1\right] \\
\beta_{2}\|z\| \leq\left(\beta_{2} / 2\right)\left[\|z\|^{2}+1\right], \quad \beta_{3}\left\|z_{f}\right\| \leq\left(\beta_{3} / 2\right)\left[\left\|z_{f}\right\|^{2}+1\right] \\
\sigma\|\tilde{\boldsymbol{W}}\|_{F} W^{*} \leq\|\tilde{\boldsymbol{W}}\|_{F}^{2}+\left(\sigma W^{*} / 2\right)^{2}
\end{gathered}
$$

Using the bounds in Eq. (A7), the time derivative $\dot{L}$ in Eq. (A6) is finally bounded as

$$
\dot{L} \leq-\kappa_{e_{m}}\left\|\boldsymbol{e}_{m}\right\|^{2}-\kappa_{z}\|\boldsymbol{z}\|^{2}-\kappa_{z_{f}}\left\|\boldsymbol{z}_{f}\right\|^{2}-\kappa_{W}\|\tilde{\boldsymbol{W}}\|_{F}^{2}+\Upsilon^{2}
$$

where $\kappa_{e_{m}}=\frac{1}{2}\left(\gamma_{1}-\gamma_{4}\right), \kappa_{z}=\frac{1}{4} q-\beta_{2} / 2-\gamma_{2}^{2} / \gamma_{1}, \kappa_{z_{f}}=\frac{1}{2}\left(q_{f}-\beta_{3}\right)$, $\kappa_{W}=\sigma-1-\gamma_{3}^{2} / \gamma_{1}-\beta_{1}^{2} / q$, and $\Upsilon^{2}=\frac{1}{2}\left[\gamma_{4}+\beta_{2}+\beta_{3}+\sigma^{2} W^{* 2} / 2\right]$. 
Define a compact set $\mathcal{B}_{C}=\left\{\zeta \in \mathcal{B}_{R} \mid\|\zeta\| \leq C\right\}$, where

$$
C=\max \left\{\sqrt{\Upsilon^{2} / \kappa_{e_{m}}}, \sqrt{\Upsilon^{2} / \kappa_{z}}, \sqrt{\Upsilon^{2} / \kappa_{z_{f}}}, \sqrt{\Upsilon^{2} / \kappa_{W}}\right\}
$$

Then $\dot{L}<0$ when $\|\zeta\|>C$. Define $\rho=\sqrt{ }\left(T_{M} / T_{m}\right) C, \mathcal{B}_{\rho}=\{\zeta \in$ $\left.\mathcal{B}_{R} \mid\|\zeta\| \leq \rho\right\}$, then $\zeta$ is uniformly ultimately bounded in $\mathcal{B}_{\rho}$ (see Ref. 29, corollary 5.1).

\section{References}

${ }^{1}$ Calise, A., Hovakimyan, N., and Idan, M., "Adaptive Output Feedback Control of Nonlinear Systems Using Neural Networks," Automatica, Vol. 37, No. 8, 2001, pp. 1201-1211.

${ }^{2}$ Hovakimyan, N., Nardi, F., Kim, N., and Calise, A., "Adaptive Output Feedback Control of Uncertain Systems Using Single Hidden Layer Neural Networks," IEEE Transactions on Neural Networks, Vol. 13, No. 6, 2002, pp. 1420-1431.

${ }^{3}$ McFarland, M. B., "Augmentation of Gain-Scheduled Missile Autopilots Using Adaptive Neural Networks," AIAA Guidance, Navigation, and Control Conference Collection of Technical Papers, Pt. 3, AIAA, Reston, VA, 1998, pp. 1786-1792.

${ }^{4}$ McFarland, M., and Stansbury, D., "Adaptive Nonlinear Autopilot for Anti-Air Missiles," AIAA Paper, Nov. 1998; also available at http://multisearch.dtic.mil, AD-A356503.

${ }^{5}$ Campa, G., Sharma, M., Calise, A., and Innocenti, M., "Neural Network Augmentation of Linear Controllers with Application to Underwater Vehicles," Proceedings of the American Control Conference, American Automatic Control Council, Evanston, IL, 2000, pp. 75-79.

${ }^{6}$ Sharma, M., and Calise, A., "Neural Network Augmentation of Existing Linear Controllers," AIAA Paper 2001-4163, Aug. 2001.

${ }^{7} \mathrm{Li}$, Y., Sundararajan, N., and Saratchandran, P., "Neuro-Controller Design for Nonlinear Fighter Aircraft Maneuver Using Fully Tuned RBF Networks," Automatica, Vol. 37, No. 8, 2001, pp. 1293-1301.

${ }^{8}$ Wohletz, J., Paduano, J., and Annaswamy, A., "Retrofit Systems for Reconfiguration in Civil Aviation," AIAA Paper 99-3964, 1999.

${ }^{9}$ Wohletz, J., Paduano, J., and Maine, T., "Retrofit Reconfiguration System for a Commercial Transport," AIAA Paper 2000-4041, 2000.

${ }^{10}$ Sastry, S., and Bodson, M., Adaptive Control: Stability, Convergence, and Robustness, Prentice-Hall, Upper Saddle River, NJ, 1989, p. 102.

${ }^{11}$ Hovakimyan, N., Yang, B.-J., and Calise, A., "An Adaptive Output Feedback Control Methodology for Non-Minimum Phase Systems," Proceedings of Conference on Decision and Control, IEEE Control System Society, Piscataway, NJ, 2002, pp. 949-954.

${ }^{12}$ Yang, B.-J., Hovakimyan, N., Calise, A., and Craig, J., "Experimental Validation of an Augmenting Approach to Adaptive Control of Uncertain Nonlinear Systems," AIAA Paper 2003-5715, 2003.
${ }^{13}$ Isidori, A., Nonlinear Control Systems, 3rd ed., Springer-Verlag, Berlin, 1995, pp. 403-416.

${ }^{14}$ Johnson, C., "Theory of Disturbance-Accomodating Controllers," Control and Dynamic Systems: Advances in Theory and Applications, edited by C. Leondes, Vol. 12, Academic Press, New York, 1976, pp. 387-487.

${ }^{15}$ King, B., and Hovakimyan, N., "An Adaptive Approach to Control of Distributed Parameter Systems," Proceedings of the Conference on Decision and Control, IEEE Control System Society, Piscataway, NJ, 2003, pp. $5715-5720$

${ }^{16}$ Johnson, E. N., and Calise, A. J., "Limited Authority Adaptive Flight Control for Reusable Launch Vehicles," Journal of Guidance, Control and Dynamics, Vol. 26, No. 6, 2003, pp. 906-913.

${ }^{17}$ Johnson, E. N., "Limited Authority Adaptive Flight Control," Ph.D. Dissertation, School of Aerospace Engineering, Georgia Inst. of Technology, Atlanta, Dec. 2000.

${ }^{18}$ Hovakimyan, N., and Calise, A., "Adaptive Output Feedback Control of Uncertain Multi-Input Multi-Output Systems Using Single Hidden Layer Neural Networks," Proceedings of the American Control Conference, American Automatic Control Council, Evanston, IL, 2002, pp. 1555-1560.

${ }^{19}$ Kim, N., "Improved Methods in Neural Network Based Adaptive Output Feedback Control with Application to Flight Control," Ph.D. Dissertation, School of Aerospace Engineering, Georgia Inst. of Technology, Atlanta, Dec. 2003.

${ }^{20}$ Bartle, R. G., The Elements of Real Analysis, 2nd ed., Wiley, NewYork, 1976, p. 163.

${ }^{21}$ Ge, S., Hang, C., Lee, T., and Zhang, T., Stable Adaptive Neural Network Control, Kluwer Academic, Boston, 2002, pp. 139-146.

${ }^{22}$ Sanner, R., and Slotine, J., "Gaussian Networks for Direct Adaptive Control," IEEE Transactions on Neural Networks, Vol. 3, No. 6, 1992, pp. 837-864.

${ }^{23}$ Hovakimyan, N., Lee, H., and Calise, A., "On Approximate NN Realization of an Unknown Dynamic System from Its Input-Output History," Proceedings of the American Control Conference, American Automatic Control Council, Evanston, IL, 2000, pp. 919-923.

${ }^{24}$ Lavretsky, E., Hovakimyan, N., and Calise, A., "Upper Bounds for Approximation of Continuous-Time Dynamics Using Delayed Outputs and Feedforward Neural Networks," IEEE Transactions on Automatic Control, Vol. 48, No. 9, 2003, pp. 1606-1610.

${ }^{25}$ Ioannou, P., and Sun, J., Robust Adaptive Control, Prentice-Hall, Upper Saddle River, NJ, 1996, p. 129.

${ }^{26}$ Ioannou, P., and Kokotović, P., Adaptive Systems with Reduced Models, Springer-Verlag, Berlin, 1983, pp. 85-88.

${ }^{27}$ Sontag, E., Mathematical Control Theory, 2nd ed., Springer-Verlag, New York, 1998, pp. 272-280.

${ }^{28}$ Kailath, T., Linear Systems, Prentice-Hall, Upper Saddle River, NJ, 1980, pp. 135-139.

${ }^{29}$ Khalil, H., Nonlinear Systems, Prentice-Hall, Upper Saddle River, NJ, 1996, p. 212. 